\title{
Das Parteiensystem vor und nach der Bundestagswahl 2013
}

von Frank Decker

Die jüngste Bundestagswahl strafte sowohl ältere Prognosen zur Entwicklung des deutschen Parteiensystems als auch die Kalküle der Parteiführungen Lügen. Weder kam es zu einer ausgeprägten Fragmentierung noch zu einer Situation, die eine strukturelle Verstetigung Großer Koalitionen gleichsam erzwingt. Im Gegenteil: Die Beteiligten standen sich in den meisten Fällen selbst im Weg, sei es aufgrund inhaltlicher oder strategischer Fehleinschätzungen, sei es infolge unkluger Personalauswahl. Jenseits spezifischer Fragestellungen, etwa mit Blick auf die Entwicklung der AfD oder die Neuaufstellung der FDP, dürfte die Entwicklung des deutschen Parteiensystems maßgeblich vom künftigen Verhältnis der drei Parteien des linken Lagers geprägt sein.

The recent federal elections refuted a number of established hypotheses on the development of the German party system and contradicted the electoral strategies of nearly all parties involved. The outcome was neither a further fragmentation of the parliamentary landscape nor the unavoidable establishment of a Grand Coalition. On the contrary: in most cases, the respective parties failed as a result of their own mistakes in selecting adequate campaign issues, strategies and/or candidates. Aside from party-specific questions, such as the trajectories of both AfD and FDP, the future of the German party system seems largely dependent upon the relationships between the three left-of-centre parties at the federal level.

\section{Fünf-, Vier- oder Sechsparteiensystem?}

Wie bereits vor vier Jahren hatten die Innenarchitekten des Berliner Reichstagsgebäudes auch nach der Bundestagswahl 2013 alle Hände voll zu tun, um die Konsequenzen des Ergebnisses zu bewältigen. In den Fraktionssaal der FDP ist die etwa halb so mitgliederstarke Fraktion der Linken eingezogen und im Plenarsaal rückt die Union zum ersten Mal seit 1949 ganz nach rechts. Waren nach 1990 stets fünf Parteien im Parlament vertreten, sind es in der kommenden Legislaturperiode nur noch vier. ${ }^{1}$ Viel hätte nicht gefehlt, dann wären es gar sechs

Anmerkung der Schriftleitung: Der Beitrag beschließt eine Reihe von Beiträgen, die sich mit der Entwicklung des deutschen Parteiensystems beschäftigt. Seit dem Heft 2/2011 kam es in diesem Rahmen zu gesonderten Untersuchungen der CSU (Heinrich Oberreuter), der CDU (Tilman Mayer), der SPD (Franz Wal- 
gewesen: Sowohl die FDP als auch die neue gegründete „Alternative für Deutschland“ (AfD) verfehlten den Einzug in den Bundestag nur knapp.

Das Scheitern beider Parteien an der Fünfprozenthürde führt dazu, dass ein hoher Anteil von Stimmen im Parlament nicht repräsentiert ist (15,8 Prozent im Vergleich zu 6,3 Prozent bei der Bundestagswahl 2009). Die Parteiensysteme auf der parlamentarischen und elektoralen Ebene klaffen dadurch auseinander. Auf der parlamentarischen Ebene halten die drei linken Parteien eine knappe Mehrheit (von umgerechnet 50,7 Prozent der Sitze gegenüber 49,3 für die Union). Diese Mehrheit hatten sie 2009 zum ersten Mal seit 1998 eingebüßt. Auf der elektoralen Ebene setzte sich dagegen der Trend von 2009 fort, indem die Achse des Parteiensystems noch einmal nach rechts verschoben wurde. Lag der zusammengenommene Stimmenanteil von Union und FDP bei der letzten Wahl bei 48,4 Prozent, so kamen die Vertreter des Mitte-Rechts-Lagers (unter Einschluss der AfD) diesmal sogar auf 51 Prozent. Der Anteil der linken Parteien (zu denen auch die Piraten gerechnet werden ${ }^{2}$ ) betrug nur 44,9 Prozent (gegenüber 47,6 Prozent im Jahr 2009).

Ein etwas anderes Bild ergibt sich, wenn man die Fragmentierung des Parteiensystems anhand der ,effektiven Parteienzahl“ berechnet. ${ }^{3}$ Hier hat die Konzentration nicht nur auf der parlamentarischen Ebene zugenommen (nur noch 3,5 effektive Parteien gegenüber 4,9 bei der Wahl 2009), sondern auch auf der elektoralen $(4,8$ gegenüber 5,6$)$. In dem letztgenannten Wert spiegelt sich der Stimmeneinbruch der beiden Volksparteien bei der Wahl vor vier Jahren, als Union und SPD zusammengenommen nur noch auf 56,8 Prozent kamen und gleichzeitig die FDP als größte der drei kleinen Parteien mehr als die Hälfte der Stimmen der kleineren der beiden großen Parteien verbuchte (14,6 gegenüber 23,0 Prozent). Für Niedermayer war dies gleichbedeutend mit einem „Typwechsel“" vom

ter/Matthias Micus), von Bündnis '90/Die Grünen (Joachim Raschke/Ralf Tils) und der FDP (Hans Vorländer). In diesem Jahr kennzeichnete Eckhart Jesse die Situation vor der Bundestagswahl, während der vorliegende Beitrag die Situation danach betrachtet.

1 CDU und CSU werden hier als eine Partei betrachtet. Von 2002 bis 2005 war die seinerzeitige PDS nur mit zwei direkt gewählten Abgeordneten vertreten, nachdem sie bei der Bundestagswahl 2002 sowohl die Fünfprozenthürde als auch den für einen Einzug in Gruppenstärke notwendigen Gewinn von drei Wahlkreismandaten verpasst hatte.

2 Die Piratenpartei lag sowohl 2009 (2,0 Prozent) als auch 2013 (2,2 Prozent) oberhalb der Schwelle, ab der von einer ,relevanten“ Partei zu sprechen ist. Sie wird deshalb in die Berechnung der Fragmentierung einbezogen.

3 Laakso, M./Taagepera, R.: „Effective Number“ of Parties. A Measure with Application to West Europe, in: Comparative Political Studies 12/1, 3-27. Der Index wird berechnet, indem man die quadrierten Stimmenanteile der (relevanten) Parteien aufsummiert und davon den Kehrwert bildet. 
System der Zweiparteiendominanz hin zu einem pluralistischen System. ${ }^{4}$ Nach derselben Typologie wäre die Bundesrepublik mit dieser Wahl wieder zum System der Zweiparteiendominanz zurückgekehrt.

Betrachtet man das Stimmenverhältnis der beiden großen Parteien, so hat sich der durch die Bundestagswahl 2009 eingeleitete Trend zur Asymmetrie fortgesetzt. Bedingt durch die Schwäche der FDP konnte die Union ihren Vorsprung vor der SPD noch einmal kräftig ausbauen (auf 15,8 gegenüber 10,8 Prozentpunkten). 2005 und 2002 lagen beide Parteien fast bzw. genau gleichauf, beim Regierungswechsel 1998 stand die SPD sogar klar vor CDU und CSU. Manche Beobachter gingen damals davon aus, dass die Sozialdemokraten ihre strukturelle Benachteiligung gegenüber der Union, die sie mit Ausnahme der „WillyWahlen" 1972 stets auf Platz zwei im Parteiensystem verwies, dauerhaft überwinden könnten. Tatsächlich entging die Partei aber bereits 2002 nur mit Glück ihrer Abwahl.

\section{Gründe für Merkels Sieg}

Auch bei der Bundestagswahl 2013 müssen, wenn man die Gründe des klaren Wahlsiegs der Union und der Niederlage der SPD bzw. von Rot-Grün verstehen will, in erster Linie politische Faktoren herangezogen werden. Ohne eine Gewichtung vorzunehmen, wären folgende Punkte zu nennen:

- $\quad$ Die Überschattung der innenpolitischen Agenda durch die Euro-Krise. Das wichtigste politische Thema, das wellenartig die gesamte Legislaturperiode prägte, bot der Opposition kaum Angriffsflächen. Einerseits trugen SPD und Grüne die Merkelsche „Rettungspolitik“ im Bundestag stets mit. Andererseits konnten und wollten sie alternative Positionen wie eine Abkehr vom strikten Sparkurs in den Südländern oder ein anderes Schuldenregime, in der Auseinandersetzung nicht forcieren, weil sie um deren Unpopularität in der Wählerschaft wussten. Die Kanzlerin nutzte unterdessen alle Möglichkeiten, sich auf der europäischen Bühne als Hüterin der nationalen Interessen in Szene zu setzen. „Mit ihrem eigentlichen Pfund, dem ,deutschen Europa', brauchte sie noch nicht einmal öffentlich zu wuchern, aber es blieb der heimliche Subtext dieser Wahlen von Anfang bis Ende.“"5

- Die gute wirtschaftliche Lage. Exzellente Wirtschaftsdaten spielen einer Regierung stets in die Hände. Weil infolge der gesunkenen Arbeitslosigkeit

4 Niedermayer, O.: Die Entwicklung des bundesdeutschen Parteiensystems, in: Decker, F./Neu, V. (Hg.), Handbuch der deutschen Parteien, 2. Aufl., Wiesbaden, 2013, 111-132.

5 Meyer, Th.: SPD - eine neue Strategie tut not, in: Neue Gesellschaft/Frankfurter Hefte 60/10 (2013), 11. 
die Angst vor einem Jobverlust in der Bundesrepublik in den letzten Jahren geringer wurde, sprudelnde Steuereinnahmen und volle Sozialkassen keine Leistungskürzungen erforderten und die Bürger durch steigende Löhne und sinkende Abgaben wieder mehr konsumierten, verfingen die Klagen über die Verwerfungen auf dem Arbeitsmarkt und die wachsende soziale Ungerechtigkeit im Lande nicht so, wie von den Oppositionsparteien erhofft. Im Gegenteil: Die Union konnte den positiven Effekt noch dadurch verstärken, dass sie die positive Entwicklung in Deutschland mit der Misere in anderen Ländern diskursiv verknüpfte.

- Die Positionierung der CDU in der politischen Mitte. Die CDU setzte den unter Merkel seit 2005 eingeschlagenen Kurs fort, sich gesellschaftspolitisch einerseits weiter zu modernisieren und andererseits in der Sozialpolitik möglichst viel linkes Ideengut zu adaptieren. Der einzig größere Rückschlag auf diesem Gebiet war die Einführung des Betreuungsgeldes, das sie sich von der Schwesterparteie CSU aufzwingen lassen musste. Die Reaktorkatastrophe im japanischen Fukushima wusste die Union dagegen in einen innenpolitischen Vorteil umzumünzen. Indem sie eine radikale Kehrtwende in der Energiepolitik vollzog, beseitigte sie nebenbei auch den wichtigsten Stolperstein für eine Zusammenarbeit mit den Grünen.

- Die Abtrennung vom Erscheinungsbild der Koalition. Die durchaus vorzeigbare Regierungsbilanz kontrastierte mit dem Bild einer Koalition, die nach katastrophalem Start auch im weiteren Verlauf der Wahlperiode nie wirklich Tritt fasste, geschweige denn harmonierte. Die Verantwortung dafür lasteten Wähler und Öffentlichkeit fast ausschließlich der FDP an, die einen beispiellosen Absturz erlebte und aus ihrem demoskopischen Tief bis zum Ende der Legislaturperiode nicht mehr herauskam. Zwar waren die Liberalen an ihrem Niedergang im Wesentlichen selbst schuld. Allerdings ist erstaunlich, dass auch andere Peinlichkeiten an Merkel und der Union scheinbar ungerührt abprallten: die Rücktritte zweier Bundespräsidenten, die die Kanzlerin selbst mit ausgewählt hatte, ein Verteidigungsminister und eine Bildungsministerin, die ihre Doktortitel nicht rechtmäßig trugen, und ein bei der nordrhein-westfälischen Wählerschaft unbeliebter Umweltminister, den Merkel gegen seinen Willen entließ.

- Die hohen Sympathiewerte der Kanzlerin. Angela Merkel ist die erste Regierungschefin, die es in Deutschland geschafft hat, während ihrer Amtszeit dauerhaft die Liste der beliebtesten Politiker anzuführen. Diese Beliebtheit verdankt sie zum einen ihrem als ausgleichend empfundenen „präsidialen“ Regierungsstil, der gleichwohl nicht als Führungsschwäche ausgelegt wird, zum anderen ihrem unprätentiösen Auftreten. Die Union hatte deshalb allen Grund, die Wahlkampagne - wie schon 2009 - ganz auf die Bundeskanzlerin zuzuschneiden.

- Die Fehler der Opposition. Die Stärke der einen ist die Schwäche der anderen. Die SPD hat nicht auf die falschen Themen gesetzt, verfügte aber in 
Peer Steinbrück über keinen optimalen Kandidaten. Der frühere Finanzminister konnte die starke Betonung der sozialen Missstände im Wahlkampf nicht glaubhaft verkörpern, zumal sie von Teilen der Wählerschaft auch als Folge sozialdemokratischer Regierungspolitik wahrgenommen wurden. Der Kampagnenstart des überstürzt ausgerufenen Spitzenmannes geriet zu einem Desaster, von dem sich die Partei erst gegen Ende des Wahlkampfs (nach dem gelungenen TV-Duell) allmählich erholte. Darüber hinaus war das personelle Angebot der SPD in der ersten Reihe zu männerlastig, um gegen die Unionszugpferde Angela Merkel und Ursula von der Leyen zu bestehen. Auch die Grünen vermochten zum Erfolg nichts beizutragen. Sie verfügten wohl nicht über die falschen Kandidaten, setzten aber statt auf Ökologie und Klimaschutz zu sehr auf diejenigen Themen (soziale Gerechtigkeit und Steuern), die auch bei der SPD und den Linken im Vordergrund standen. Damit verbaute man sich zugleich die Chancen für ein mögliches schwarzgrünes Regierungsbündnis. Darüber hinaus wurde die Partei kurz vor der Wahl mit nicht aufgearbeiteten Sünden ihrer eigenen Vergangenheit (Pädophilie-Debatte) konfrontiert, was ihre Kampagne vollends aus dem Tritt brachte.

Aufschlussreich ist ein Blick auf die - wegen ihrer Ungenauigkeit freilich umstrittene - Wählerwanderungsbilanz. ${ }^{6}$ Die SPD verdankte ihre bescheidenen Zugewinne bei der Wahl hauptsächlich der Schwäche von Grünen und Linken, von denen sie zusammen 920.000 Stimmen erhielt. Aus dem Mitte-Rechts-Lager gab es ebenfalls ein leichtes Plus von 140.000 Stimmen, da im Saldo mehr Wähler von der FDP zur SPD überliefen, als diese an Union und AfD verlor. Der Hauptgrund des schwachen Wahlergebnisses lag in der nicht gelungenen Mobilisierung von Nichtwählern, worauf auch die kaum gestiegene Wahlbeteiligung (von 70,8 auf 71,5 Prozent) hindeutet: Die Bürger, die der SPD 2009 massenhaft den Rücken gekehrt hatten, blieben diesmal erneut zu Hause oder wechselten zur Union; diese konnte im Ergebnis dreimal so viele vormalige Nichtwähler rekrutieren wie die SPD (1.130.000 gegenüber 360.000).

Die geringe Mobilisierungsfähigkeit der SPD dürfte nicht nur darauf zurückzuführen sein, dass Themen und Kandidat ihre Wirkung verpassten, sie war auch Ausdruck der fehlenden Machtperspektive. Die von der Partei angestrebte rotgrüne Koalition gelangte im Wahljahr laut Umfragen zu keinem Zeitpunkt in die Nähe einer eigenen Mehrheit. Je näher der Wahltermin rückte, desto größer wurde der Abstand zu Union und FDP. Sozialdemokraten und Grüne hatten sich von ihren Erfolgen in den alten Ländern blenden lassen, wo es ihnen dank des bei

6 Vgl. hierzu die Daten von Infratest dimap. 
Landtagswahlen üblichen Zwischenwahleffektes und der Zurückdrängung der Linken gelungen war, gleich vier schwarz-gelbe durch rot-grüne bzw. grün-rote Regierungen abzulösen. Dass dieses Szenario auf die Bundesebene nicht übertragbar sein würde, hätte bereits ein Blick auf die näheren Umstände dieser Erfolge zeigen müssen. ${ }^{7}$ So aber landeten die Sozialdemokraten durch das Festhalten am unrealistischen Wahlziel Rot-Grün am Ende in derselben misslichen Situation wie 2009, als sie nicht mehr auf Sieg, sondern nur noch auf Platz spielen konnten.

\section{Die Entwicklung des Parteiensystems seit 2005}

\section{Die Linke: gesamtdeutsche Kraft oder ostdeutsche Regionalpartei?}

Die Aussichtslosigkeit von Rot-Grün ist eine Folge der Segmentierung des „linken Lagers“. Mit der Entstehung der gesamtdeutschen Linkspartei trat die Entwicklung des deutschen Parteiensystems 2005 in eine neue Phase. ${ }^{8}$ Der Übergang von der Viereinhalb- zur Fünfparteienstruktur wurde möglich, nachdem sich in den alten Ländern eine Abspaltung von der SPD gebildet und diese mit der ostdeutschen PDS zur Partei „Die Linke“ fusioniert hatte. Die Gründung der „Wahlalternative Arbeit \& soziale Gerechtigkeit“ (WASG) erfolgte aus Protest gegen die von der rot-grünen Bundesregierung unter Kanzler Gerhard Schröder betriebenen Arbeitsmarkt- und Sozialreformen. Ihr lag also keine neue Konfliktlinie zugrunde, sondern die Kritik, dass sich die Herkunftspartei von ihrer traditionellen Position auf der sozialökonomischen Achse zu weit entfernt habe. Symbolhaft markiert wurde dies durch die Person des früheren SPDVorsitzenden Lafontaine, dessen Übertritt zur WASG eine wesentliche Voraussetzung für den Erfolg der Partei in den alten Bundesländern darstellte.

Auch nach dem Abgang Lafontaines musste die Linke um ihre Position als fünfte Partei nicht fürchten. Das organisatorische Fundament der im Osten bestens vernetzten PDS und die neue Aktualität von Verteilungsfragen gaben ihr gute Chancen, auf dem 2009 erreichten Niveau zu verharren. Die gleichzeitige Bedienung der regionalistischen und sozialökonomischen cleavages versprach eine

7 In Baden-Württemberg (2011) verdankte er sich dem zufälligen Zusammentreffen mehrerer begünstigender Faktoren (Fukushima, Stuttgart 21, unpopulärer CDU-Ministerpräsident), in NordrheinWestfalen (2012) gelang er erst auf dem Umweg über eine geduldete rot-grüne Minderheitsregierung, in Schleswig-Holstein (2012) musste der Südschleswigsche Wählerverband zusätzlich in die Koalition aufgenommen werden und in Niedersachsen (2013) reichte es für Rot-Grün am Ende nur dank weniger hundert Stimmen.

8 Vgl. Decker, F.: Die Zäsur, in: Berliner Republik 7/6 (2005), 66-71. 
stabile Wählerkoalition, obwohl die Partei durch die Westausdehnung ihre reine Ost-Identität verlor. Symptomatisch dafür ist, dass sich die Wählerzusammensetzung auch in den neuen Ländern in Richtung der sozial benachteiligten Gruppen verschiebt. ${ }^{9}$ Dennoch geriet das Projekt der gesamtdeutschen Linken nach der Bundestagswahl rasch ins Stocken. ${ }^{10}$ War die Linke noch 2011 in sieben von zehn Landtagen der alten Bundesrepublik vertreten, scheiterte sie bei den Wahlen in Schleswig-Holstein (2012), Nordrhein-Westfalen (2012) und Niedersachsen (2013) an der Fünfprozenthürde. Damit drohte die Partei in die Rolle zurückzufallen, die sie ab 2005 eigentlich überwinden wollte, nämlich die einer (reinen) Regionalpartei des Ostens.

Ursächlich für diese Entwicklung waren (und sind) vor allem hausgemachte Schwierigkeiten. Die wenig professionell agierenden Landesverbände in den alten Ländern, das wechselseitige Misstrauen zwischen der neuen West- und der alten Ost-Linken sowie ständige Querelen in der Parteiführung vermittelten der Öffentlichkeit den Eindruck, dass die Partei in erster Linie mit sich selbst beschäftigt sei. ${ }^{11}$ Hinzu traten schwierige Umfeldbedingungen. Die Überlagerung der innenpolitischen Agenda durch die Euro-Krise machte es der Linken schwer, Gegenposition zu beziehen. Öffentlich vernehmbarer (und Resonanz versprechender) Widerstand gegen die von Regierung und SPD/Grünen gemeinsam vertretene Krisenpolitik kam und kommt heute eher von „rechts“ - auch aus Teilen des Regierungslagers. Dies lässt sich zugleich an der Wählerwanderung ablesen: Während in den alten Bundesländern die abtrünnigen Wähler der Linken überwiegend bei der SPD landeten bzw. zu ihr zurückkehrten, liefen sie im Osten scharenweise zur AfD über, die dort ihre besten Ergebnisse erzielte.

Wenn sich die Verluste dennoch in Grenzen hielten, lag das in erster Linie am unerwartet guten Abschneiden in den alten Ländern. Zusammen mit dem früheren West-Berlin trugen diese mehr als die Hälfte der Stimmen (52,6 Prozent) zum Gesamtstimmenergebnis der Linken bei. Der Abwärtstrend bei den Landtagswahlen konnte so gestoppt werden. Ursächlich dafür war einerseits die Schwäche von SPD und Grünen, andererseits die verbesserte Außendarstellung, nachdem das überforderte Führungsduo Klaus Ernst und Gesine Lötzsch im Juni

9 Vgl. Niedermayer, O.: Die Wählerschaft der Linkspartei.PDS 2005: Sozialstruktureller Wandel bei gleichbleibender politischer Positionierung, in: Zeitschrift für Parlamentsfragen 37/3 (2006), 523-538.

10 Vgl. Decker, F.: Das Verhältnis der SPD gegenüber der LINKEN - Die offene Zukunft, in: Hirscher, G./Jesse, E. (Hg.), Extremismus in Deutschland, Baden-Baden, 2013, 549-563.

11 Vgl. Neu, $V$ :: Der gestoppte Aufstieg? Perspektiven der Linken, in: Jesse, E./Sturm, R. (Hg.), „Superwahljahr“ 2011 und die Folgen, Baden-Baden, 2012, 133-151. 
2012 durch Bernd Riexinger und Katja Kipping abgelöst wurde. Gleichzeitig verfügte die Linke mit Gregor Gysi und Sahra Wagenknecht über zwei medienversierte Aushängeschilder, die in der Auseinandersetzung mit der Konkurrenz überzeugten. In Hessen, wo ihr bei der zeitgleich mit der Bundestagswahl stattfindenden Landtagswahl der Wiedereinzug in das Parlament gelang, nutzte der bundespolitische Aufwind in den alten Ländern der Partei gleich doppelt. Als größte Oppositionspartei im Bundestag (noch vor den Grünen) könnte sie an diesen Trend anknüpfen, wenn die Wähler ihre Unzufriedenheit mit der wieder aufgelegten Großen Koalition demnächst erneut einseitig bei der SPD abladen.

\section{Die Piratenpartei: der kurze Frühling einer Protestpartei?}

Zum unverhofften shooting star des Parteiensystems wurde in der abgelaufenen Wahlperiode - vorübergehend - die Piratenpartei. ${ }^{12}$ Beginnend mit der Berliner Abgeordnetenhauswahl im September 2011, gelang es der erst 2006 gegründeten neuen Gruppierung in kurzer Folge, vier Mal hintereinander in ein Landesparlament einzuziehen. Nachdem die „Piraten“ bei der Bundestagswahl 2009 und allen nachfolgenden Landtagswahlen lediglich ein schmales Kernwählersegment von etwa zwei Prozent erreicht hatte, war die Öffentlichkeit zunächst geneigt, ihren Berliner Sensationserfolg als Ausreißer abzutun und auf die dort besonders günstigen lokalen Bedingungen zurückzuführen. Mit den weiteren Erfolgen im Saarland, in Nordrhein-Westfalen und in Schleswig-Holstein musste diese Einschätzung korrigiert werden. Die „Piraten“ sprachen, wie die Wahlanalysen zeigten, offensichtlich nicht nur die netzaffinen Themenwähler an, die die Positionen der etablierten Parteien in diesem Feld als hoffnungslos rückständig empfanden. Sie fungierten jetzt auch als Sammelbecken von Unzufriedenen und Protestwählern, die der Politik und ihrer herkömmlichen Vertretern generell überdrüssig waren.

Der Höhenflug in den Umfragen, der die Partei nach der Euphorie der Landtagswahlerfolge im Frühsommer 2012 zeitweilig in den zweistelligen Bereich katapultierte, währte jedoch nur kurz. Als Protestpartei hatten die „Piraten“ offenkundig in hohem Maße von der Verunsicherung der Bevölkerung im Zuge der Euro-Krise profitiert. Nachdem sie anfangs darauf vertrauen konnten, dass von ihnen abseits der Netzpolitik keine Antworten auf die anstehenden politischen

12 Vgl. u.a. Bieber, Ch./Leggewie, C. (Hg): Unter Piraten. Erkundungen in einer neuen politischen Arena, Bielefeld, 2012; Hensel, A./Klecha, St./Walter, F.: Meuterei auf der Deutschland. Ziele und Chancen der Piratenpartei, Berlin, 2012; und Niedermayer, O. (Hg.): Die Piratenpartei. Wiesbaden, 2013. 
Probleme erwartet würden, erwies sich die programmatische Unbestimmtheit in der Außendarstellung zunehmend als Problem. Den nur durch das Metathema Partizipation und Transparenz zusammengehaltenen Neuankömmlingen fiel die Entwicklung gemeinsamer inhaltlicher Positionen sichtlich schwer. Zugleich verstrickten sie sich in Widersprüche. Ihre Aktivisten mussten lernen, dass politische Führung in Partei und Fraktion ohne ,geschützte Räume“ nicht funktioniert. Das Transparenzziel wurde durch die Anonymisierung und Pseudonymisierung im Internet Lügen gestraft. Zudem war die Partizipationsbereitschaft der PiratenMitglieder keineswegs größer als bei den anderen Parteien. Die Selbstentzauberung verstärkte nicht nur die innerparteilichen Konflikte, sondern führte auch dazu, dass die Medien, die den Aufstieg der neuen Partei zunächst fast ausnahmslos mit unverhohlener Sympathie begleitet hatten, ihr zunehmend kritischer begegneten.

Symptomatisch für das Scheitern der Piraten war ihre Unfähigkeit, aus der NSAAbhöraffäre politischen Nutzen zu ziehen. Der Skandal, der durch Enthüllungen des früheren US-Geheimdienstmitarbeiters Edward Snowden ein halbes Jahr vor der Bundestagswahl plötzlich über die deutsche Innenpolitik hereinbrach, hätte dem Neuankömmling eigentlich beste Mobilisierungschancen bieten müssen. Er spielte im Wahlkampf aber ebensowenig eine Rolle wie andere Fragen der Netzpolitik oder das Partizipationsthema.

\section{Die AfD: Ende der Schwäche des parteiförmigen Rechtspopulismus?}

Warum es in der Bundesrepublik nicht zur flächendeckenden Etablierung einer rechtspopulistischen oder -extremistischen Partei gekommen ist, bleibt in gewisser Weise ein Rätsel. ${ }^{13}$ Fragmentierungstendenzen des nationalen Lagers mündeten zwar in den achtziger Jahren in eine „dritte Welle“ des Rechtsextremismus. Auf ihr zogen die 1983 als Abspaltung von der CSU entstandenen Republikaner (REP) drei Mal, die 1987 gegründete Deutsche Volksunion (DVU) des - 2013 verstorbenen - Münchener Verlegers Gerhard Frey neun Mal und die NPD vier Mal in Landesparlamente ein. Die letztgenannte Partei wurde bereits 1964 ins Leben gerufen, konnte aber nach Wahlerfolgen in der zweiten Hälfte der sechziger Jahre erst wieder im Jahre 2004 an ihre damalige Stärke anknüpfen, nachdem sie das Bundesland Sachsen gezielt zur Hochburg auszubauen wusste. Inzwischen hat die gegenüber früheren Jahrzehnten deutlich radikalisierte NPD die

13 Decker, F.: Die Erfolglosigkeit des parteiförmigen Rechtspopulismus in Deutschland - Bedingungen und Gründe, in: Hirscher, G./Jesse, E. (Hg.), Extremismus in Deutschland, Baden-Baden, 2013, 63-78. 
Führungsrolle im rechtsextremen Lager übernommen, während die einst so verheißungsvoll gestarteten Republikaner nur noch ein Schattendasein fristen. Von einem Durchbruch auf der nationalen Ebene bleibt der harte Extremismus der NPD weit entfernt - bei der Bundestagswahl 2013 fiel die Partei gegenüber ihrem schwachen Ergebnis von 2009 noch einmal um 0,2 auf 1,3 Prozentpunkte zurück. $^{14}$

Ein Grund für diese Schwäche liegt gerade im Extremismus der NPD, der auf viele Wähler abschreckend wirkt und die Entwicklung einer populistischen Strategie der Wähleransprache vereitelt. Allerdings sind in der Bundesrepublik auch ideologisch gemäßigtere Gruppierungen, die eine solche Strategie verfolgten, bisher nicht zum Erfolg gekommen. Weder gelang der Versuch, eine bereits bestehende Partei auf rechtspopulistische Pfade zu führen, wie dem verstorbenen FDP-Politiker Jürgen W. Möllemann unterstellt wurde, noch waren Neugründungen wie die Hamburger Statt-Partei, der Bund Freier Bürger oder die SchillPartei in der Lage, ihre Anfangserfolge zu wiederholen und über die regionale Ebene auszudehnen. ${ }^{15}$

Mit der „Alternative für Deutschland“ schickt sich jetzt eine neue Gruppierung an, die Geschichte der Erfolglosigkeit des Rechtspopulismus in der Bundesrepublik zu beenden. Die im April 2013 gegründete Partei, die vom Hamburger VWL-Professor Bernd Lucke angeführt wird, verfehlte den Einzug in den Bundestag nur knapp. Auch in Hessen kam sie bei der Landtagswahl aus dem Stand auf vier Prozent der Stimmen. Ob sie weiter zulegen und sich im Parteiensystem festsetzen kann, wird vor allem von zwei Dingen abhängen: der Resonanz des von ihr in den Mittelpunkt gerückten Euro-Themas und der Fähigkeit, mit den restriktiven Bedingungen umzugehen, die das Aufkommen und den Erfolg rechtspopulistischer Parteien in der Bundesrepublik in der Vergangenheit erschwerten.

Die AfD wird nicht zu Unrecht als single-issue-Partei apostrophiert. Neben der Auflösung der Währungsunion vertritt sie zwar eine Reihe von anderen Forderungen, die an programmatische Gewinnerformeln des Rechtspopulismus in Westeuropa anknüpfen und zu einer breiteren Plattform ausgebaut werden könnten. Dazu zählen u.a. die Einführung direktdemokratischer Elemente und veränderte Regeln für die Einwanderung. Diese Positionen werden aber nur schlagwortartig erwähnt und nicht weiter ausgeführt. Umso detailfreudiger ist das 
Programm bei der Kernforderung, der ,geordneten Auflösung“ der Währungsunion. Der Euro soll entweder durch die alten nationalen Währungen oder die Schaffung kleiner Währungsverbünde (Nord- und Süd-Euro) ersetzt werden. Um dies zu erreichen, postuliert die Partei als ersten Schritt die Einführung nationaler Parallelwährungen zum Euro in den südeuropäischen Ländern, deren verbindlicher Anteil am bargeldlosen Zahlungsverkehr bei 50 Prozent liegen soll und danach sukzessive zu steigern sei. Mit der Rückkehr zu den nationalen Währungen sollen die Unterschiede in der Wettbewerbsfähigkeit ausgeglichen werden, die ein Funktionieren der Währungsunion in der Vergangenheit unmöglich gemacht hätten. Damit ließe sich auch die Gefahr eines Auseinanderbrechens der gesamten Union bannen.

Dass diese Thesen durchaus verfangen und von relevanten Bevölkerungsgruppen unterstützt werden, kann man exemplarisch auf den Leserbriefseiten der Qualitätszeitungen studieren. Aufschlussreich ist ein Vergleich zur Sarrazin-Debatte vor drei Jahren, wo ein - auf den ersten Blick - ähnliches Phänomen sichtbar wurde. Im Gegensatz zur heutigen Euro-Diskussion ist Sarrazins Abrechnung mit der bundesdeutschen Zuwanderungspolitik ${ }^{16}$ aber erstaunlich verpufft und parteipolitisch folgenlos geblieben. Warum? Eine mögliche Antwort könnte die unterschiedliche ideologische Gefechtslage liefern. Multikulturalismuskritik ist, obwohl sie in diesem Fall von einem früheren SPD-Politiker artikuliert wurde, überwiegend im konservativen und rechten Spektrum beheimatet. Dass sie auch in Teilen der linken Wählerschaft auf Zustimmung trifft, steht dem nicht entgegen - es entspricht vielmehr dem kulturellen Traditionalismus der Arbeiterklasse, den der amerikanische Soziologe Seymour Martin Lipset schon vor über fünfzig Jahren diagnostizierte.

Die Forderung nach Auflösung der Währungsunion ist demgegenüber nicht zwangsläufig eine konservative oder rechte Position. Sie wird von sozialdemokratischen Vordenkern geteilt ${ }^{17}$ und findet, angestoßen durch Äußerungen Oskar Lafontaines, auch in der Linkspartei Widerhall. So gesehen ist es bemerkenswert, dass das Führungspersonal der AfD fast ausschließlich aus dem liberalkonservativen Lager stammt. Blickt man auf die Mitglieder und Wähler, geht die Unterstützung dagegen quer durch alle Parteien und Schichten. So konnte die Partei bei der Bundestagswahl annähernd so viele Stimmen aus dem linken La-

16 Sarrazin, Th.: Deutschland schafft sich ab. Wie wir unser Land aufs Spiel setzen, Berlin, 2010.

17 Vgl. u.a. Scharpf, F.W.: Rettet Europa vor dem Euro, in: Berliner Republik 14/2 (2012), 52-61; Streeck, $W .:$ Gekaufte Zeit. Die vertagte Krise des demokratischen Kapitalismus, Berlin, 2013. 
ger (von SPD und Linken) zu sich herüberziehen wie aus dem bürgerlichen Lager von Union und FDP (520.000 gegenüber 720.000). Einzig die Grünen waren mit lediglich 90.000 Abwanderern gegen die euroskeptische Stimmung weitgehend immun.

Es bleiben allerdings Zweifel, ob die Kritik am Euro in der Bevölkerung tatsächlich so stark zündet, wie die AfD hofft. Die gemeinsame Währung ist bei den Bürgern ja durchaus geschätzt; dasselbe gilt für den Unternehmensbereich, der aufgrund ausbleibender Kursschwankungen über eine erweiterte Planungssicherheit verfügt. Was die Menschen vor allem umtreibt, ist die Stabilität der Währung und die Sorge, dass Deutschland für die Schulden anderer Mitglieder der Euro-Zone aufkommen muss. Wie die hohen Zustimmungsraten zum Krisenmanagement der Regierung und speziell der Bundeskanzlerin zeigen, fühlen sie sich in dieser Frage von der Politik keineswegs schlecht vertreten. Dass sich die Bundesrepublik durch die Anleihekäufe der Europäischen Zentralbank de facto bereits in einer Haftungsunion befindet, mochte die Regierung verständlicherweise nicht offen zugeben. Auch die rot-grüne Opposition hielt sich an dieser Stelle lieber bedeckt, um dem Verdacht vorzubeugen, sie würde eine noch stärkere Vergemeinschaftung der Schulden (etwa durch die Ausgabe von Eurobonds) anstreben.

Bleibt die Frage, ob die neue Partei in der Lage sei, die sich ihr bietenden Gelegenheiten zu nutzen. Das größte Hindernis für den Erfolg rechtspopulistischer Gruppierungen in der Bundesrepublik liegt in der ihnen drohenden Stigmatisierung, die zugleich ihren Zugang zu den meinungsbildenden Medien erschwert. Auch bei der AfD waren die Kritiker mit dem Vorwurf des Rechtspopulismus schnell bei der Hand. Bisher hat sich die Partei dagegen relativ glaubhaft verwahren können. Unter dem Strich profitiert sie davon, dass sie eher ein liberalbürgerliches als ein populistisches Profil pflegt. Zwar verzeichnet die AfD bis jetzt noch keine prominenten Überläufer aus dem Lager von Union und FDP. Allerdings macht sich gerade in der Union die Sorge breit, dass von der Partei eine Attraktion auf konservativ eingestellte Teile der Wählerschaft ausgehen könnte, die mit dem unter Merkel eingeschlagenen Modernisierungskurs hadern - von der Schulpolitik über den Atomausstieg bis hin zu Homoehe und Frauenquote. Obwohl diese Themen in der Programmatik der neuen Partei nachrangig sind, könnten sie im Hinterkopf der Wähler eine wichtige Rolle spielen.

Gefahren drohen der AfD vor allem von innen. An der Frage, wie man mit unerwünschten Unterstützern von rechtsaußen umgeht, sind in der Bundesrepublik bisher noch alle Etablierungsversuche einer rechtspopulistischen oder -konser- 
vativen Kraft gescheitert. Gerade gemäßigt auftretende Gruppierungen werden von solchen Unterstützern gerne als Trittbrett genutzt, um der Stigmatisierung als rechtsextrem oder -populistisch zu entgehen. Bestrebungen, die Programmatik der AfD für eine breitere populistische Plattform zu öffnen, sind in einigen Landesverbänden bereits erkennbar und haben $\mathrm{zu}$ ersten Absatzbewegungen geführt. ${ }^{18}$ Die Parteispitze versucht dem durch eine möglichst straffe, zentralistische Führung entgegenzutreten, der allerdings durch die rechtlichen Regelungen (im Parteiengesetz) enge Grenzen gesetzt sind und die zugleich an der Parteibasis auf Widerspruch trifft. Ob der AfD ein kontrollierter Aufbau der Organisation gelingt, ohne dass ihr öffentliches Bild und die notwendige Geschlossenheit im Auftreten nach außen Schaden nimmt, ist also keineswegs ausgemacht.

Immerhin: Das Risiko, das Abschneiden bei der Bundestagswahl und der am selben Tag stattfindenden Landtagswahl in Hessen durch ein schwaches Landtagswahlergebnis in Bayern zu gefährden (wo eine Woche früher gewählt wurde), konnte die AfD umgehen, indem sie auf die Kandidatur in Bayern nach heftiger interner Debatte verzichtete. Auch der Wahlkalender spricht dafür, dass sich die politische Konkurrenz auf weitere Erfolge der neuen Partei einstellen muss. So erscheint ihr Einzug bei den Wahlen zum Europäischen Parlament im Mai 2014 fast sicher: Zum einen gilt dort - wenn überhaupt ${ }^{19}$ - nur eine DreiProzent-Sperrklausel, zum anderen gibt es keine bessere Bühne, um antieuropäischen Protest auszudrücken. Und bei den Landtagswahlen in Brandenburg, Sachsen und Thüringen im Herbst könnte die AfD erneut davon profitieren, dass die Bereitschaft zur Protestwahl in den ostdeutschen Ländern stärker ausgeprägt ist als in den westdeutschen.

\section{Der Niedergang der Liberalen}

$\mathrm{Zu}$ den Ironien des Bundestags-Wahlergebnisses gehört, dass Union und FDP mit einem bequemen Vorsprung durchs Ziel gegangen wären, wenn beide Seiten in der Woche vor der Wahl nicht die Nerven verloren hätten. Ihr Desaster bei der bayerischen Landtagswahl veranlasste die Liberalen zu einer panischen Leihstimmenkampagne, die eine ebenso heftige Gegenwehr der Union provozierte.

18 „Populist in Teilzeit“, in: Der Spiegel, Nr. 43 vom 21.Oktober, 2013, 40.

19 Nachdem das Bundesverfassungsgericht die bei den Wahlen zum Europäischen Parlament bislang gültige Fünf-Prozent-Klausel im November 2011 ,gekippt“ hatte, beschloss der Bundestag im Juni 2013, eine Drei-Prozent-Klausel einzuführen. Ob diese mit dem Urteil vereinbar ist, steht in Zweifel und wird von Karlsruhe vermutlich noch vor der Wahl erneut geprüft. 
Diese wollte ihren Fehler bei der Niedersachsenwahl im Januar 2013 unter keinen Umständen wiederholen, wo sie das Buhlen um die Zweitstimmen zugelassen hatte und dadurch am Ende die Regierungsmehrheit verlor. Merkels Annahme, die Liberalen würden den Einzug aus eigener Kraft schaffen, erwies sich als folgenschwerer Irrtum.

Das Drama der letzten Wahlkampfwoche wirft ein bezeichnendes Licht auf den Umgang, den CDU und CSU mit der FDP über die gesamte Legislaturperiode hinweg pflegten. Weil die Union den Steuersenkungen, die die Liberalen vollmundig angekündigt hatten (und für deren Umsetzung bis zur verlorenen Landtagswahl in Nordrhein-Westfalen ein ausreichendes Zeitfenster bestand), schon in den Koalitionsverhandlungen einen Riegel vorschob, war deren Absturz aus großer Fallhöhe vorprogrammiert. ${ }^{20}$ Machtstrategisch handelte es sich dabei nicht gerade um ein kluges Vorgehen, es sei denn, der Umstieg in eine andere Koalition wäre von vornherein beabsichtigt gewesen. Dafür gibt es freilich keine Anhaltspunkte. Umso verwunderlicher ist es, wie wenig das Schicksal ihres angeblichen Wunschkoalitionspartners den Unionsparteien am Herzen lag.

Die Ursachen der FDP-Misere reichen bis weit vor 2009 zurück, als die Partei mit 14,6 Prozent ihr bestes Ergebnis bei einer Bundestagswahl erreichte. ${ }^{21}$ Die Unterscheidung zwischen dem angeblich guten Oppositions- und schlechten Regierungspolitiker Guido Westerwelle stimmte schon damals nicht. Sie verkennt, dass die einseitig marktliberale Ausrichtung der Partei und ihre koalitionspolitische Fixierung auf die Union von ihrem damaligen Vorsitzenden maßgeblich befördert wurden. Unter Westerwelle tappte die FDP mit ihrer gebetsmühlenhaft vorgetragenen Forderung nach einem ,einfacheren, gerechteren und niedrigeren Steuersystem“ zudem geradewegs in die Populismusfalle.

Als schwerer Fehler erwies sich zudem Westerwelles Entscheidung, statt des Finanzministeriums das vermeintlich prestigeträchtigere Auswärtige Amt für die Partei zu reklamieren. ${ }^{22}$ Während der geborene Innenpolitiker dort vergeblich versuchte, an die stolze Tradition seiner liberalen Vorgänger Walter Scheel und Hans-Dietrich Genscher anzuknüpfen, konnte sich die Union im Finanzressort

20 Vgl. Decker, F.: Wenn die Populisten kommen. Beiträge zum Zustand der Demokratie und des Parteiensystems, Wiesbaden, 2013, $105 \mathrm{ff}$.

21 Ebd., $110 \mathrm{ff}$.

22 Vgl. Vorländer, H.: Welche Koalition sichert das Überleben? Bündnisaussichten der FDP, in: Decker, F./Jesse, E. (Hg), Die deutsche Koalitionsdemokratie vor der Bundestagswahl 2013, Baden-Baden, 2013, $394 \mathrm{ff}$. 
als Gestalterin der Euro-Krisenpolitik wählerwirksam profilieren. Auch in der Rechts- und Gesellschaftspolitik ließ die FDP jegliche Akzente vermissen.

Das Ausscheiden aus dem Bundestag stellt die Partei unter ihrem neuen Vorsitzenden Christian Lindner vor eine extrem schwierige Herausforderung. Als außerparteiliche Opposition wird es ihr an medialer Präsenz schmerzlich fehlen. Wieweit sich dies durch die Arbeit in den Ländern (wo sie noch in neun Parlamenten und einer Regierung vertreten ist) und auf der kommunalen Ebene wettmachen lässt, bleibt abzuwarten. Gefahr droht auch von der veränderten Akteursund Wettbewerbssituation. Das Auftreten der AfD führt dazu, dass die Liberalen ihr bisheriges Alleinstellungsmerkmal als einzige bürgerliche Kraft neben der Union eingebüßt haben. Die Versuchung, der FDP mit wirtschaftsliberalen Positionen ihr Terrain streitig zu machen, dürfte für die AfD naheliegen. In der Kombination von nationalen, gesellschaftspolitisch konservativen und euroskeptischen ${ }^{23}$ Positionen würde sie damit die bereits angesprochene programmatische „Gewinnerformel“ oder -bürgerliche Parteien in Europa typisch ist. Dass zumindest die letztgenannten (euroskeptischen) Positionen auch in der FDP weit verbreitet sind, hat das Ergebnis ihres Mitgliederentscheids vom Dezember 2011 gezeigt, als immerhin 44 Prozent der Abstimmenden der von der Parteiführung befürworteten Einrichtung des dauerhaften Europäischen Stabilitätsmechanismus (ESM) widersprachen.

\section{Die erwartbare Neuauflage der Großen Koalition}

Die Neuauflage der Großen Koalition war zu Beginn des Wahlkampfes wahrscheinlicher als an dessen Ende. Dass Schwarz-Gelb nochmals die Chance auf eine klare Mehrheit haben würde, ließ sich kaum vorausahnen. ${ }^{24}$ Am Wahlabend stand für kurze Zeit sogar die Möglichkeit einer absoluten Mehrheit der Union im Raum. Bei einem Vorsprung von lediglich ein oder zwei Mandaten wäre dies für Merkel freilich ein Horrorszenario gewesen. Auch so entpuppte sich das Ergebnis als „Pyrrhussieg“. Die Unionsparteien zogen zwar mit Abstand die meisten Stimmen auf sich, waren aber auf einen Koalitionspartner aus dem anderen politischen Lager angewiesen. Weil SPD und Grüne sich zierten, wurden

23 Zum Euroskeptizismus vgl. Hartleb, F.: A Thorn in the Side of European Elites: The New Euroscepticism, Brüssel, 2011.

24 Vgl. Decker, F./Jesse, E.: Koalitionslandschaft im Wandel? Eine Einführung, in: dies. (Hg.), Die deutsche Koalitionsdemokratie vor der Bundestagswahl 2013, Baden-Baden, 2013, 22 ff. Die dort enthaltenen Prognosen wurde etwa ein halbes Jahr vor der Wahl abgegeben. 
auch Überlegungen in Richtung einer unionsgeführten Minderheitsregierung angestellt oder baldige Neuwahlen erwogen - allerdings mehr von außen als von den Akteuren selbst. Beiden Parteien war bewusst, dass an der Bildung einer stabilen Mehrheitsregierung kein Weg vorbei führte; am Ende musste also eine von ihnen bereit sein, mit Merkels Union zu koalieren.

Die Argumente, die im Vorfeld für eine größere Wahrscheinlichkeit der schwarz-roten (statt schwarz-grünen) Koalition sprachen, sollten sich nach der Wahl bewahrheiten. In Teilen der Union und der konservativen Presse gab es während der Sondierungsphase starke Sympathien für Schwarz-Grün. Diese dürften jedoch hauptsächlich von der Interessenlage der Union diktiert gewesen sein, das heißt der Aussicht, mit den durch ihr schlechtes Wahlergebnis empfindlich getroffenen Grünen einen „leichteren“ Partner zu bekommen als mit der SPD. Das Scheitern der schwarz-grünen Sondierung wurde in den meisten Kommentaren vor allem der grünen Seite angelastet, der im entscheidenden Moment der Mut gefehlt habe, es mit der Union zu probieren. Aus Sicht der Grünen gab es dafür freilich gute Gründe. Die Partei konnte ja ihr Wahlprogramm in möglichen Koalitionsverhandlungen nicht einfach zur Disposition stellen, auch wenn dessen dezidiert linke Ausrichtung zum schwachen Wahlresultat beigetragen hatte. Dasselbe galt für ihr Spitzenpersonal. Ob Schwarz-Grün bei einem besseren Wahlergebnis der Grünen wahrscheinlicher geworden wäre, bleibt eine müßige Spekulation. Deren Führung hätte es unter diesen Bedingungen sicher leichter gehabt, der Basis eine solche Koalition zu vermitteln. Für die Union wäre es im Gegenzug aber schwieriger geworden: Sie hätte in Verhandlungen mit starken Grünen mehr Zugeständnisse machen und am Ende auch einen Jürgen Trittin als Minister akzeptieren müssen.

Bei den Sozialdemokraten war die Bereitschaft, erneut in eine Große Koalition einzutreten, an der Parteispitze zunächst stärker ausgeprägt als unter den Funktionären und an der Basis. Dafür gibt es mindestens drei Gründe: Erstens winken die Pfründe einer Regierungsbeteiligung stets nur dem Führungspersonal. Zweitens wog in der Parteispitze die Sorge, bei einer Koalition von Union und Grünen die letzteren als strategischen Partner mittel- und langfristig zu verlieren, schwerer als die Hoffnung auf künftige Wahlerfolge in der Opposition. Und drittens wusste man in der Führung besser als an der Basis, dass die SPD 2009 nicht an ihrer Regierungsbeteiligung in der Großen Koalition gescheitert war, „sondern an ihrer Verfassung außerhalb dieser Regierung.“25 
Empirische Untersuchungen der bisherigen Großen Koalitionen auf Bundes- und Länderebene zeigen, dass diese keineswegs immer nur dem größeren Partner zum Vorteil gereichen. So wie es der SPD im Bund 1969 gelang, von der Juniorrolle in der Großen Koalition in eine kleine Koalition (mit der FDP) unter ihrer Führung umzusteigen, so konnte sie auch in Mecklenburg-Vorpommern (1998) und in Berlin (2001) die CDU als führende Regierungspartei verdrängen - in beiden Fällen mit Hilfe der PDS. Nur wo sie hinter der CDU deutlich zurücklag - wie in Baden-Württemberg (1996), Thüringen (1999) oder Sachsen (2009) -, musste sie nach ihrer Regierungsbeteiligung auf die Oppositionsbänke wechseln.

Wie Umfragen zeigen, bewerten die Anhänger der SPD inzwischen eine Große Koalition keineswegs negativ; die Zahl der Unterstützer ist mit etwa zwei Dritteln genauso groß wie unter den Unionsanhängern. Dies scheint zu dem von manchen SPD-Politikern kolportierten Eindruck einer überwiegenden Ablehnung der Großen Koalition an der Basis nicht ganz zu passen. Dass die Vorbehalte vor allem aus den Ländern kommen, die von der SPD (mit)regiert werden, dürfte nicht nur mit der Furcht vor Niederlagen bei anstehenden Kommunal- und Landtagswahlen zu tun haben. Es verweist auch auf die unterschiedliche Interessenlage zwischen den dortigen Landesverbänden und der Bundes-SPD. Für die erstgenannten wäre das Verbleiben in der Opposition im Zweifel komfortabler. Denn dann stünden die SPD-regierten Länder mit ihrer Mehrheit im Bundesrat der unionsgeführten Bundesregierung als geschlossene Phalanx gegenüber. Sie könnten diese unter Druck setzen, die Finanzausstattung der Länder zu verbessern. Regiert die SPD dagegen in einer Großen Koalition mit, müssen sich die von Hannelore Kraft angeführten Landesfürsten zugleich mit der Bundespartei und den von der SPD gestellten Ministern arrangieren, die qua Amt automatisch stärker dem Bundesinteresse verpflichtet sind.

Wechselt man von der parteilichen oder innerparteilichen zur gesamtstaatlichen Interessenperspektive, spricht gerade mit Blick auf den Föderalismus vieles für die Neuauflage der Großen Koalition. Die Einführung der Schuldenbremse, das Auslaufen des Solidarpakts im Jahre 2019 und die Infragestellung des geltenden Finanzausgleichssystems durch die reichen Bundesländer im Süden machen eine grundlegende Neuordnung der Finanzbeziehungen notwendig. Außerdem könnte eine Große Koalition das Kooperationsverbot in der Bildungspolitik lockern, das

schliss die SPD allein vier (!) Vorsitzende. Fast ein Jahr hielt sie die Republik 2008 mit dem YpsilantiDebakel in Atem, das am Ende auch Kurt Beck das Amt kostete. Dieser warf den Vorsitz im September 2008 vor laufenden Kameras hin, nachdem ihn Frank-Walter Steinmeier und Franz Müntefering bei der Entscheidung über die Kanzlerkandidatur desavouierten. 
sie selbst 2006 eingeführt hatte. Union und SPD haben zwar keine eigene Mehrheit im Bundesrat, könnten diese aber erreichen, wenn es in zwei weiteren Bundesländern zu schwarz-roten Bündnissen käme. (Eine schwarz-grüne Regierung hätte demgegenüber nur auf die sechs Stimmen des CSU-regierten Bayern zählen können.) Auch mit Blick auf die europäischen Herausforderungen ist es kein Schaden, das bisherige informelle Zusammenwirken der beiden Volksparteien in eine förmliche Zusammenarbeit zu überführen.

Offenkundig sind dagegen die von einer Großen Koalition ausgehenden Demokratieschäden. Union und SPD kontrollieren im Bundestag zusammen vier Fünftel der Mandate. Wesentliche Kontrollrechte, wie die Einsetzung eines Untersuchungsausschusses oder eine Normenkontrollklage, sind von den beiden verbliebenen Oppositionsparteien mangels Masse nicht einsetzbar. Letzteres könnte etwa zur Folge haben, dass das Prüfungsrecht des Bundespräsidenten stärker ins Spiel kommt, wie es bereits bei der vorangegangenen Großen Koalition der Fall war. ${ }^{26}$ Eine solche Entwicklung erscheint nicht unbedingt wünschenswert. Genauso wenig ist es angängig, den Einsatz der Minderheitenrechte allein vom goodwill der Mehrheit abhängig zu machen. Union und SPD sollten deshalb bereit sein, die Quoren im Rahmen einer förmlichen Verfassungsänderung anzupassen. ${ }^{27}$

\section{Perspektiven der Parteien- und Koalitionslandschaft mit Blick auf 2017}

Aus normativer Sicht sind Große Koalitionen nicht erstrebenswert. In bestimmten politischen Situationen können sie zwar sinnvoll sein, generell sollten sie aber die Ausnahme bleiben und nur als Übergangslösung fungieren, bis das in der parlamentarischen Demokratie übliche Alternierungsprinzip wiederhergestellt ist. Wenn es nach vier Jahren einer (kleinen) schwarz-gelben Koalition jetzt zur Neuauflage der Konstellation von 2005 kommt, zeigt das, dass die Voraussetzungen für eine solche Wiederherstellung im deutschen Parteiensystem noch nicht vorliegen. Schon 2005 war die Mehrzahl der journalistischen wie der wissenschaftlichen Beobachter davon ausgegangen, dass die Ära des klassischen

26 Vgl. Decker, F.: Regieren im „Parteienbundesstaat“. Zur Architektur der deutschen Politik, Wiesbaden, 2011, $334 \mathrm{f}$.

27 Die naheliegendste Lösung wäre eine Absenkung auf 20 Prozent. Oder man belässt es beim 25-ProzentQuorum, sieht aber zusätzlich vor, dass ein Verfahren auch beantragt werden kann, wenn ihm mindestens zwei Fraktionen beitreten. Der letztere Vorschlag erscheint mit Blick auf die möglichen Regierungskonstellationen im Vielparteiensystem zukunftsfester. 
Volksparteien-Dualismus, die in der Bundesrepublik das Standardmodell der kleinen Zweierkoalition begründet hatte, unwiderruflich ans Ende gelangt sei. Zwei Zukunftsszenarien - ein negatives und ein positives - wurden damals ausgemalt. Entweder - so hieß es - komme es wie in Österreich zu einer Perpetuierung der Großen Koalition. Oder eine multiple Koalitionslandschaft wie in den skandinavischen Ländern würde entstehen, in der lagerübergreifende Dreierbündnisse das Bild prägten. ${ }^{28}$

Beide Szenarien haben sich nicht bewahrheitet. Auf der Bundesebene zeigte das Wahlergebnis 2009, dass Mehrheiten für kleine Zweierkoalitionen weiter möglich waren - allerdings nur im bürgerlichen Lager. Und auf der Länderebene blieb die Koalitionslandschaft zwischen Ost- und Westdeutschland segmentiert. Der Hauptgrund dafür liegt in der unterschiedlichen Position der Linken. In den neuen Bundesländern hat deren Stärke einen doppelten Effekt: Einerseits sorgt sie dafür, dass Mehrheiten für kleine Koalitionen schwer zu erlangen sind. Andererseits lässt sie rot-rote Koalitionen nur zu, wenn die SPD stärker als die Linke ist und in der Koalition den Ministerpräsidenten stellen kann. Die letztgenannte Bedingung ist etwa in Thüringen und Sachsen-Anhalt nicht gegeben, weshalb diese Länder heute (genauso wie Mecklenburg-Vorpommern und Berlin) von schwarz-roten ${ }^{29}$ Koalitionen regiert werden.

In den alten Ländern bleibt die Linke dagegen $\mathrm{zu}$ schwach, um die Bildung schwarz-gelber oder rot-grüner Koalitionen dauerhaft zu vereiteln. Deshalb gibt es hier in der Regel keine Notwendigkeit, Große Koalitionen zu bilden oder andere lagerübergreifende Zweier- bzw. Dreierbündnisse einzugehen. Wo solche Bündnisse zustande kamen (Ampelkoalition in Brandenburg 1990 bzw. Bremen 1991, Schwarz-Grün in Hamburg 2008 und Schwarz-Gelb-Grün im Saarland 2009), zerbrachen sie allesamt vor Ablauf der Legislaturperiode.

Eine vergleichbare Situation wie im Bund gab es bislang nur in Hessen (2008 und 2013) sowie in Nordrhein-Westfalen (2010). Hier konnten bzw. können SPD und Grüne ohne die Linke keine Regierungsmehrheit erreichen. In den Ländern ist die SPD bereit, diese Machtoption zu nutzen. Auf der Bundesebene, wo sie eine Koalition bisher ausgeschlossen hat, wird sie dasselbe tun und sich für eine Zusammenarbeit mit der Linken öffnen müssen, wenn sie eine Chance haben will, die Union als führende Regierungspartei 2017 abzulösen. Gelingt es, die

28 Vgl. Decker, F.: Veränderungen in der Parteienlandschaft, in: Der Bürger im Staat 59/2 (2009), 74-81.

29 Die Bezeichnung Große Koalition ist diesem Fall falsch, weil es sich nicht um ein Zusammengehen der beiden stärksten Parteien handelt. 
Segmentierung im linken Lager zu überwinden, stünden die Zeichen im deutschen Parteiensystem auf Rückkehr zur Bipolarität. Zwei annähernd gleich starke, koalitionspolitisch abgrenzbare Formationen würden dann um die Regierungsmacht streiten. Die Situation wäre ähnlich der in den achtziger Jahren, nur dass sich das linke Lager jetzt statt aus zwei aus drei Teilen zusammensetzte. ${ }^{30}$

Gegen ein solches Szenario spricht die Ungewissheit, wie sich das Verhältnis von SPD, Grünen und Linken entwickeln wird. Einerseits ist das Interesse an einer gemeinsamen Machtperspektive noch kein Garant, dass sich bestehende personelle und programmatische Differenzen überwinden lassen. Andererseits führen die unterschiedlichen Koalitionsmöglichkeiten der beteiligten Partner dazu, dass ihre strategischen Interessen nicht deckungsgleich sind. Einer vollständigen Vereinnahmung im linken Lager dürften sich insbesondere die Grünen widersetzen. Denn hält sich die Öko-Partei den Weg frei, gegebenenfalls auch mit den Unionsparteien zu paktieren, könnte sie demnächst eine ähnliche Züngleinrolle im Parteiensystem einnehmen wie früher die FDP. Warum sollte sie auf diesen Vorteil verzichten? Wo sie die Koalitionsfrage offen hielten, hat das den Grünen elektoral in der Regel nicht geschadet. Auch links eingestellte Delegierte und Mitglieder lassen sich vom Schwenk in das bürgerliche Lager durchaus überzeugen, wenn er von der Führung gut vorbereitet und begründet wird.

Eine vollständige Bipolarisierung wird und kann es wohl allein schon aufgrund der föderalen Verfassung der Bundesrepublik nicht geben. Dass sich Landespolitiker der Koalitionsräson ihrer Bundesparteien bisweilen entziehen, gehört hierzulande zu den normalen Usancen der Koalitionspolitik. Wenn Parteien, die auf Bundesebene gegeneinander stehen, in den Ländern miteinander regieren, stößt das antagonistische Modell notgedrungen an Grenzen. Das Gegenüber zweier klar unterscheidbarer Blöcke mag zwar unter Demokratiegesichtspunkten vorzugswürdig sein, indem es dem Wähler eine ebenso klare Entscheidung ermöglicht. Fraglich ist aber, ob es die faktischen Entscheidungsnotwendigkeiten und alternativen im komplizierten Regierungsgeschehen noch hinreichend abbildet.

30 Vgl. Spier, T.: Realisierbare Koalitionsoption im Zeithorizont 2013/2017? Perspektiven von Rot-RotGrün, in: Decker, F./Jesse, E. (Hg.), Die deutsche Koalitionsdemokratie vor der Bundestagswahl 2013, Baden-Baden, 2013, 369-388. 